\title{
Composition Chimique Et Activité Antimicrobienne De L'huile Essentielle De Porophyllum Ruderale (Jacq.) Cass. (Asterales ; Asteraceae) Récoltée En Côte d'Ivoire
}

\author{
Kouassi Kouamé Séraphin, \\ Kouame Bosson Antoine, \\ Mamyrbékova-Békro Janat Akhanovna, \\ Bekro Yves-Alain
}

Laboratoire de Chimie Bio-Organique et de Substances Naturelles (LCBOSN) - UFR-SFA Université Nangui Abrogoua Abidjan Côte d'Ivoire

Doi:10.19044/esj.2020.v16n27p268 URL:http://dx.doi.org/10.19044/esj.2020.v16n27p268

Résumé

L'huile essentielle de la partie aérienne de Porophyllum ruderale (Jacq.) Cass. (Asterales ; Asteraceae) obtenue par entraînement à la vapeur a été analysée par Chromatographie en Phase Gazeuse couplée à la Spectrométrie de Masse. L'activité antimicrobienne de l'huile essentielle a été évaluée sur des bactéries et champignons de référence du Centre Suisse de Recherches Scientifiques en Côte d'Ivoire. L'huile essentielle est constituée essentiellement de monoterpènes $(94,69 \%)$, d'hydrocarbures aromatiques $(4,39 \%)$ et de sesquiterpènes $(0,27 \%)$. Les composés majoritaires sont $\beta$ myrcène $(35,52 \%), \gamma$-terpinène $(26,37 \%), \beta$-pinène $(21,65 \%)$, limonène $(9,26 \%)$ et 1 -undécène $(4,39 \%)$. L'huile essentielle a inhibé la plupart des souches testées, Klebsiella pneumoneae, Bacillus subtilus CIP, Staphylococcus auréus, Staphylococcus épidermidis, Candida albicans, Candida tropicalis et Candida glabrata, avec une concentration minimale inhibitrice (CMI) de 3,75mg/ml. Cependant, elles ne possèdent aucun effet sur Salmonella typhimirium SO 66, Eschérichia coli ATCC 25922 et Pseudomonas aéruginosa ATCC 27853.

Cette étude a revélé la composition chimique de l'huile essentielle de la partie aérienne de $P$. ruderale. Elle a en outre montré le bon potentiel antimicrobien de l'huile essentielle.

Mots clés : Porophyllum Ruderale, Huile Essentielle, Activité Antimicrobienne, Côte d'Ivoire 


\title{
Chemical Composition And Antimicrobial Activity Of The Essential Oils Of Porophyllum Ruderale (Jacq.) \\ Cass. (Asterales ; Asteraceae) Harvested In Côte d'Ivoire
}

\author{
Kouassi Kouamé Séraphin, \\ Kouame Bosson Antoine, \\ Mamyrbékova-Békro Janat Akhanovna, \\ Bekro Yves-Alain
}

Laboratoire de Chimie Bio-Organique et de Substances Naturelles (LCBOSN) - UFR-SFA Université Nangui Abrogoua Abidjan Côte d'Ivoire

Abstract

Essential oil of the aerial part of Porophyllum ruderale (Jacq.) Cass. (Asterales; Asteraceae) obtained by steam distillation was analyzed by Gas Chromatograhy coupled to Mass Spectroscopy. Then the antimicrobial activity of essential oil was evaluated on reference bacteria and fungi from the Swiss Center for Scientific Research in Côte d'Ivoire. Essential oil consists mainly of monoterpenes $(94.69 \%)$, aromatic hydrocarbons $(4.39 \%)$ and sesquiterpenes $(0.27 \%)$. The major compounds are $\beta$-myrcene $(35.52 \%), \gamma$ terpinene (26.37\%), $\beta$-pinene $(21.65 \%)$, limonene $(9.26 \%)$ and 1-undecene (4.39\%). Essential oil inhibited most of the strains tested, Klebsiella pneumoneae, Bacillus subtilus CIP, Staphylococcus aureus, Staphylococcus epidermidis, Candida albicans, Candida tropicalis and Candida glabrata, with a minimum inhibitory concentration (MIC) of $3.75 \mathrm{mg} / \mathrm{ml}$. However, they have no effect on Salmonella typhimirium SO 66, Escherichia coli ATCC 25922 and Pseudomonas aeruginosa ATCC 27853.

This study revealed the chemical composition of the essential oil of Porophyllum ruderale. It also showed the good antimicrobial potential of this essential oil.

Keywords : Porophyllum Ruderale, Essential Oil, Antimicrobial Activity, Côte d'Ivoire

\section{1-Introduction}

La flore ivoirienne présente une grande biodiversité qui profite à la médecine traditionnelle endogène en Côte d'Ivoire. Dans cette partie de l'Afrique subsaharienne, le recours à la phytothérapie aromatique est une 
pratique médicale récurrente du fait de la valeur intrinsèque des plantes aromatiques et médicinales. Au nombre de ces espèces végétales utiles, Porophyllum ruderale trouve une place de choix. C'est une plante annuelle érigée, généralement plutôt peu ramifiée, atteignant 20 à $100 \mathrm{~cm}$ de hauteur. Elle est plus connue sous le nom de "coriandre bolivienne" ou de "arnicabrésilienne", et est originaire d'Amérique Tropicale (Fonseca et al., 2006). En Côte d'Ivoire, une soudaine apparition et prolifération de cette adventice sur les parcelles agricoles est observée. Cette plante se propage principalement par les graines. Elle appartient à la famille botanique des Asteraceae, et produit beaucoup de semences avec un taux de germination élevé (Dogba et al., 2018). En Amérique latine, plusieurs études ont été menées sur la plante. Des phénols totaux et une activité antioxydante ont été revélés dans des extraits par extraction ultrasons de la partie aérienne de $P$. Ruderale (Conde-Hernández et al., 2014). Des dérivés de thiophène isolés de $P$. ruderale présenteraient une forte activité contre les formes promastigotes et amastigotes axéniques de Leishmania amazonensis (Takahashi et al., 2011). Peu d'études chimiques existent en Côte d'Ivoire sur la plante. Vues les limites de certains produits de synthèse face à la recrudescence de certaines pathologies, le recours à des produits naturels contre plusieurs souches de référence s'avère nécessaire. C'est pour le renforcement de la recherche pour une meilleure connaissance de la composition phytochimique et du potentiel biologique de $P$. ruderale, qu'a été initié la présente étude dont le but a été de caractériser l'huiles essentielles. puis d'évaluer son activité antibactérienne sur des souches de référence.

\section{2- Materiel Et Methodes}

\section{1- Matériel Végétal}

La partie supérieure de la plante fraiche a été récoltée au début $\mathrm{d}^{\prime}$ Octobre dans la commune de Bingerville $\left(5^{\circ} 21^{\prime} \mathrm{N}, 3^{\circ} 53^{\circ} \mathrm{O}\right)$ une ville de Côte d'Ivoire, située au bord de la lagune Ébrié, et appartenant au District Autonome d'Abidjan. La plante a été identifiée par un technicien botaniste du Centre Suisse de Recherches Scientifiques en Côte d'Ivoire (CSRS, Adiopodoumé - Côte d'Ivoire) et authentifié au Centre National de Floristique (CNF) d'Abidjan au moyen de l'herbier existant sous le numéro UCJ018336.

\section{2- Extraction de I'huile essentielle}

La technique de distilltion par entraînement à la vapeur à l'aide d'un dispositif en acier inoxidable à quatre compartiments a été utilisée pour extraitre l'huile essentielle de la matrice végétale (MV). La chaudière (60 1 de capacité) est reliée à une grande cuve par un tuyau en inox. La grande cuve (hauteur: $100 \mathrm{~cm}$, diamètre interne: $51 \mathrm{~cm}$ soit un volume de $0,2 \mathrm{~m}^{3}$ ) contient quatre grilles solidaires d'une tige amovible. Sur les grilles, a été déposée la 
partie aérienne $(5,5 \mathrm{~kg})$ de MV. De cette cuve, la vapeur d'eau entraîne les composés volatils dans une troisième cuve (hauteur : $100 \mathrm{~cm}$, diamètre interne : $41 \mathrm{~cm}$ soit un volume de $0,13 \mathrm{~m}^{3}$ ) qui sert de réfrigérant. L'HE sont obtenue dans un quatrième compartiment servant de système de récupération. L'huile essentielle est mise dans des piluliers emballés dans du papier alluminium puis conservée dans un congélateur à environ $4^{\circ} \mathrm{C}$.

\section{3- Analyse de l'huile essentielle}

L'analyse de l'HE diluée dans le dichlorométane $(1: 100)$ a été réalisée sur un chromatographe GC (7890A, Agilent Technologies) couplé à un spectromètre de masse (5975C, Agilent Technologies). Un échantillon de $1^{\circ} \mathrm{HE}(1 \mu \mathrm{l})$ a été injecté dans une colonne capillaire $\mathrm{HP}-5 \mathrm{MS}$ à $250^{\circ} \mathrm{C}$. La température du four a été programmée à $40^{\circ} \mathrm{C}$ pendant $5 \mathrm{~min}$, ensuite à $2^{\circ} \mathrm{C} /$ min pendant 15 min jusqu'à $250^{\circ} \mathrm{C}$, avec un débit de $10^{\circ} \mathrm{C} / \mathrm{min}$ jusqu'à $300^{\circ} \mathrm{C}$. L'hélium a été utilisé comme gaz porteur avec un débit de $1 \mathrm{ml} / \mathrm{min}$. Le détecteur MS avait une température de $280^{\circ} \mathrm{C}$ et une tension de $1,4 \mathrm{kV}$ Etaient détectables les ions dont le rapport masse/charge est compris entre 40 à 500. L'identification des composés a été effectuée par comparaison des indices de rétention, calculés à partir des temps de rétention et des spectres de masse obtenues avec celles de la base de données de l'Institut National des Normes et de la Technologie (NIST) et de la littérature (Paolini, 2005; Daouda, 2015).

\section{4- Evaluation de l'activité antibactérienne de l'huile essentielle \\ 2.4.1- Mesure des diamètres des zones d'inhibition}

Les souches de référence (Staphylococcus auréus CIP 4.8, Staphylococcus épidermidis CIP.53124, Salmonella typhimirium SO 66, Eschérichia coli ATCC 25922, Klébsiella pneumoneae, Pseudomonas aéruginosa ATCC 27853 , Bacillus subtulis ATCC 6633, Candida albicans ATCC 10231 , Candida tropicalis ATCC 13803 et Candida glabrata ATCC 66032) utilisées pour les études de sensibilité vis-à-vis des différentes concentrations de l'HE extraite proviennent du laboratoire du Centre Suisse de Recherches Scientifiques en Côte d'Ivoire. Dans les mêmes conditions, les zones d'inhibition des antibiotiques Gentamycine et l'Amphotéricine B sont mésurées pour le contrôle. L'activité antibactérienne des différentes concentrations de $1^{`} \mathrm{HE}$ a été déterminée contre chaque souche bactérienne par la méthode de Berghe et Vlietinck (Berghe et Vlietinck, 1991). L'ensemencement de l'inoculum de $1 \mathrm{ml}$ a été réalisé avec une culture de 18 à $20 \mathrm{~h}\left(10^{5}-10^{6} \mathrm{UFC} / \mathrm{ml}\right)$, en surface du milieu Mueller Hinton $(\mathrm{MH})$ préalablement coulé dans des boîtes de Pétri. Après $15 \mathrm{~min}$, des puits ont été découpés à l'aide de pipettes Pasteur. Le fond des puits a été obturé par une goutte de gélose $\mathrm{MH}$ pour limiter la diffusion des huiles sous la gélose. 
Ensuite, $50 \mu \mathrm{d}$ d'HE à différentes dilutions, ont été distribués dans chaque puits. Après diffusion, les cultures ont été incubées dans des étuves à $37^{\circ} \mathrm{C}$ pendant $24 \mathrm{~h}$. Les auréoles d'inhibition ont été mesurées avec un pied à coulisse.

\subsection{2- Détermination des concentrations minimales}

Dans $10 \mathrm{ml}$ de bouillon $\mathrm{MH}$, sont repiqués $0,1 \mathrm{ml}$ de bouillon pour les Eschérichia coli, Salmonella, Bacillus, klébsiella, candida et 0,3 ml pour Staphylococcus aureus et épidermidis. Ces cultures sont incubées à $37{ }^{\circ} \mathrm{C}$ pendant 3 à 5 h jusqu'à l'apparition d'une légère opalescence d'environ $510^{7}$ bactéries/ml. Ensuite, $1 \mathrm{ml}$ de ces bouillons est ajouté à $10 \mathrm{ml}$ de bouillon $\mathrm{MH}$ préalablement chauffé à $37^{\circ} \mathrm{C}$ (inoculum). Puis, $100 \mu \mathrm{l}$ de la solution d'extrait de $\mathrm{HE}$ dans la colonne $\mathrm{n}^{\circ} 12$ de la microplaque, $50 \mu \mathrm{l}$ de bouillon $\mathrm{MH}$ à partir de la colonne $\mathrm{n}^{\circ} 11$ jusqu'à la colonne $\mathrm{n}^{\circ} 2$ et $100 \mu \mathrm{l}$ de bouillon $\mathrm{MH}$ dans la colonne $\mathrm{n}^{\circ} 1$. Une dillution est faite à partir de la colonne $\mathrm{n}^{\circ} 12$ jusqu'à la colonne $\mathrm{n}^{\circ} 3$ en prélevant $50 \mu \mathrm{l}$ à chaque fois, et une distribution de $50 \mu 1$ de $l^{\prime}$ inoculum dans chaque puits de la colonnes $n^{\circ} 12$ à $n^{\circ} 2$. Enfin, la lecture à l'œil nu est faite après une incubation à $37^{\circ} \mathrm{C}$ pendant $18 \mathrm{~h}$.

\section{3- Resultats Et Discussion}

\section{1-Rendement Et Composition Phytochimique De L'Huiles Essentielle}

L'HE de la partie aérienne de $P$. ruderale a été extraite par entraînement à la vapeur $24 \mathrm{~h}$ après la récolte. L'huile est liquide, de couleur jaune, et extraite avec un rendement d'environ $0,10 \pm 0.02 \%$. Ce rendement pourrait être attribué à plusieurs facteurs dont la technique de distillation, la période et la zone de récolte, a été calculé par le quotient de la masse de 1'HE extraite à la masse de la matière végétale distillée.

L'HE a été analysée par CPG/SM. L'identification des constituants a été réalisée à l'aide des indices de rétention et des spectres de masse. Les indices de rétention ont été calculés à partir des temps de rétentions (Kovats, 1958; IUPAC, 1997). Outre les monoterpènes (94,69\%), les hydrocarbures aromatiques $(4,39 \%)$, des sesquiterpènes $(0,12 \%)$, huit $(08)$ composés faisant environ 99,20\% de la composition totale de l'HE (Tableau 1), sont représentés par un hydocarbure oléfinique (1-undécène, 4,39\%), un monoterpène acyclique insaturé minoritaire ( $\beta$-ocimène, $0,49 \%$ ), un sesquiterpène tricyclique ( $\alpha$-copaène, $0,27 \%)$, un monoterpène acyclique majoritaire ( $\beta$-myrcène, 35,52\%), un monoterpène cyclique isomérique ( $\gamma$ terpinène, 26,37\%), deux monoterpènes bicycliques isomères ( $\alpha$-pinène, $1,40 \%$ et $\beta$-pinène, $21,65 \%$ ) et un hydrocarbure terpénique cyclique (limonène, $(9,26 \%)$. 
Tableau 1: Principaux constituants l'HE de $P$. ruderale identifiés par GPG/SM et leurs pourcentages.

\begin{tabular}{cccccc}
\hline pic & Composés & TR $(\min )$ & IR & $\mathrm{m} / \mathbf{z}$ & $\%$ HE \\
\hline 1 & $\alpha$-Pinène & 12,7 & 926 & 136 & 1,40 \\
\hline 2 & $\boldsymbol{\beta}$-Pinène & 15,5 & 969 & 136 & $\mathbf{2 1 , 6 5}$ \\
\hline 3 & $\boldsymbol{\beta}$-Myrcène & 16,9 & 991 & 136 & $\mathbf{3 5 , 5 2}$ \\
\hline 4 & Limonène & 19,2 & 1025 & 136 & $\mathbf{9 , 2 6}$ \\
\hline 5 & $\beta$-Ocimène & 20,2 & 1038 & 136 & 0,49 \\
\hline 6 & $\boldsymbol{\gamma}$-Terpinène & 20,9 & 1048 & 136 & $\mathbf{2 6 , 3 7}$ \\
\hline 7 & 1-Undécène & 24,0 & 1092 & 154 & 4,39 \\
\hline 8 & $\alpha$-Copaène & 43,5 & 1379 & 204 & 0,27 \\
\hline \multicolumn{3}{c}{ Monoterpènes } \\
\hline Sesquiterpènes & & & 94,69 \\
\hline \multicolumn{5}{c}{ Tydrocarbures aromatiques } \\
\hline
\end{tabular}

TR: Temps de Rétention; IR: Indice de Rétention; m/z: masse sur charge

$\mathrm{Au}$ regard des résultats obtenus, il est clair que la technique d'extraction utilisée n'influencerait pas la composition chimique des HEs, mais aurait un impact sur le rendement d'extraction. En effet, les mêmes constituants ont été identififiés par Raggi et al.(2014) dans l'HE obtenue avec un rendement différent du nôtre, par hydrodistillation des parties aériennes de deux sous-espèces cultivées de Porophyllum ruderale à savoir Porophyllum ruderale subsp. macrocephalum (PRM) et Porophyllum ruderale subsp. ruderale (PRR).

La composition chimique de l'HE de $P$. ruderale semble présenter des variations entre les stades de croissance de la plante (végétatif et reproducteur) (Raggi et al.,2014).

Par ailleurs en Bolivie en revanche, Loayza et al. (2000) ont identifié le Sabinène (64\%) comme carbure monoterpénique majoritaire. Au Brésil, Fonsceca et al. (2006) ont montré la présence de $\beta$-phéllandrène majoritaire dans les HE des feuilles et des fleures de la même plante. Enfin Au Venezuela, Rondón et al. (2008) ont montré la coexistence dominante du binôme limonène- $\beta$-phellandrène $(50,3 \%)$ et du sabinène $(20,2 \%)$.

3.2- Activités antimicrobiennes de l'huile essentielle

L'activité antimicrobienne a été estimée au regard du diamètre de la zone d'inhibition microbienne, et ce, en se référant à un standard antibactérien ou antifongique connu. selon Berghe et Vlietinck (1991), l'activité antibactérienne est nulle pour un diamètre de la zone d'inhibition (d.i.) inférieur ou égale à $8 \mathrm{~mm}$; faible pour d.i. compris entre 8 et $14 \mathrm{~mm}$, moyenne pour d.i. compris entre 14 et $20 \mathrm{~mm}$; forte pour d.i. supérieur ou égal à 20 $\mathrm{mm}$. Les résultats des tests antimicrobiens (Tableau 2) ont montré que 1'HE de $P$. ruderale est dépourvue d'activité contre Salmonella typhimirium $S O$ 66, Eschérichia coli ATCC 25922 et Pseudomonas aéruginosa ATCC 27853, 
mais sont moyennement efficaces contre Klebsiella pneumoneae, Bacillus subtilus CIP, Staphylococcus auréus, Staphylococcus épidermidis, Candida albicans, Candida tropicalis et Candida glabrata. Elle présente par ailleurs, un effet batériostatique avec une plus grande sensibilité contre Staphylococcus épidermidis CIP.53124 et Klébsiella pneumoneae. Par ailleurs des analyses antibactériennes de 1'HE de cette espèce faites par Rondón et al. (2008) contre les bactéries à Gram positif (Staphylococcus auréus, Enterococcus faecalis) et à Gram négatif (Escherichia coli, Klebsiella pneumoniae, Pseudomonas aeruginosa), ont montré un large spectre d'activité avec des valeurs minimales de concentration inhibitrice (CMI) allant de 20 à $200 \mu \mathrm{g} / \mathrm{mL}$.

Tableau 2 : Concentrations minimales inhibitrice et bactéricide des huilles essentielles de $P$. ruderale.

\begin{tabular}{|c|c|c|c|c|c|c|c|c|}
\hline \multirow[t]{2}{*}{ Souches } & \multicolumn{4}{|c|}{ HE P.ruderale } & \multicolumn{4}{|c|}{ Gentamycine (référence) } \\
\hline & $\begin{array}{l}\mathrm{Di} \\
(\mathrm{mm})\end{array}$ & $\begin{array}{l}\text { CMI } \\
(\mathrm{mg} / \mathrm{mL})\end{array}$ & $\begin{array}{l}\text { CMB } \\
(\mathrm{mg} / \mathrm{mL})\end{array}$ & $\begin{array}{l}\mathrm{CMB} / \\
\mathrm{CMI}\end{array}$ & $\begin{array}{l}\mathrm{Di} \\
(\mathrm{mm})\end{array}$ & CMI & CMB & $\begin{array}{l}\text { CMB } \\
\text { /CMI }\end{array}$ \\
\hline $\begin{array}{l}\text { Staphylococcus } \\
\text { auréus CIP } 4.83\end{array}$ & 15 & 3,75 & 30 & 8 & 30 & 0,005 & 0,01 & 2 \\
\hline $\begin{array}{l}\text { Staphylococcus } \\
\text { épidermidis } \\
\text { CIP.53124 } \\
\end{array}$ & 24 & 3,75 & 15 & 4 & 30 & $\begin{array}{l}0,002 \\
5\end{array}$ & 0,005 & 2 \\
\hline $\begin{array}{l}\text { Salmonella } \\
\text { typhimirium SO } \\
66\end{array}$ & 0 & - & - & - & 28 & - & - & - \\
\hline $\begin{array}{l}\text { Eschérichia coli } \\
\text { ATCC } 25922\end{array}$ & 0 & - & - & - & 28 & - & - & - \\
\hline $\begin{array}{l}\text { Klébsiella } \\
\text { pneumoneae }\end{array}$ & 20 & 3,75 & 15 & 4 & 35 & 0,005 & 0,01 & 2 \\
\hline $\begin{array}{l}\text { Pseudomonas } \\
\text { aéruginosa } \\
\text { ATCC } 27853 \\
\end{array}$ & 0 & - & - & - & 30 & - & - & - \\
\hline $\begin{array}{l}\text { Bacillus subtulis } \\
\text { ATCC } 6633\end{array}$ & 18 & 3,75 & 30 & 8 & 34 & $\begin{array}{l}0,002 \\
5\end{array}$ & 0,005 & 2 \\
\hline & & & & & Amph & éricine & (référer & \\
\hline $\begin{array}{l}\text { Candida albicans } \\
\text { ATCC } 10231\end{array}$ & 14 & 3,75 & 30 & 8 & 35 & 0,05 & 0,1 & 2 \\
\hline $\begin{array}{l}\text { Candida } \\
\text { tropicalis ATCC } \\
13803\end{array}$ & 12 & 3,75 & 30 & 8 & 30 & 0,05 & 0,1 & 2 \\
\hline $\begin{array}{l}\text { Candida glabrata } \\
\text { ATCC } 66032\end{array}$ & 13 & 3,75 & 30 & 8 & 35 & 0,05 & 0,1 & 2 \\
\hline
\end{tabular}

Di :diamètre d'inhibition ; CMI : Concentration Minimale Inhibitrice ; CMB : Concentration Minimale Bactéricide

\section{Conclusion}

La composition chimique de l'HE de $P$. ruderale récoltée en Côte d'Ivoire, est constituée essentiellement de monoterpènes. Les composés 
majoritaires sont $\beta$-myrcène $(35,52 \%), \quad \gamma$-terpinène $(26,37 \%)$, et $\beta$ pinène $(21,65 \%)$. L'activité antimicrobienne de cette huile est moyenne contre Klebsiella pneumoneae, Bacillus subtilus CIP, Staphylococcus aureus, Staphylococcus epidermidice, Candida albicans, Candida tropicalis et Candida glabrata et pourrait ouvrir de nouvelles pistes de recherche contre la résistance antimicrobienne.

\section{Remerciements :}

Les auteurs remercient Gwaenael Chamoulaud et Galyna Shul de l'Université du Quebec à Montréal pour les analyses chromatographiques ; Téré Henry technicien botaniste pour l'identification de la plante et Touré Sadikou technicien du Laboratoire d'analyse pour les analyses antimicrobiennes, au Centre Suisse de Recherches Scientifiques en Côte d'Ivoire (CSRS, Adiopodoumé -Côte d'Ivoire).

\section{References :}

1. Berghe V.D.A., Vlietinck A.J. (1991). Screening methods for antibacterial and antiviral agents from higher plants. Methods in plant biochemistry. Hostettman, K Ed., London: Academic press.

2. Daouda T. (2015). Etudes chimique et biologique des huiles essentielles de quatre plantes aromatiques médicinales de Côte d'Ivoire. Chimie organique. Université Felix Houphouet Boigny, Côte d'Ivoire, p154.

3. Dogba M., Malan D.F., Neuba D.F.R., 2 et Konan A.S. (2018). Biologie et écologie de Porophyllum ruderale (Jacq.) Cass.,une Compositae nouvellement apparue en Côte d'Ivoire. Journal of Animal \& Plant Sciences,36(3), p5907-5918.

4. Fonseca, M. C. M., Meira, R. M. S. A., \&Casali, M. D. V. (2006). Anatomie d'organes végétatifs et histolocalisation des lipides et des composés phénoliques dans Porophyllum ruderale (Asteraceae). Planta Daninha, 24(4), p707-13.

5. IUPAC. (1997). Compedium of Chemical Terminology, 2è édition (le Livre d'or). Wilkinson Blackwell Scientific Publications, Oxford. P108.

6. Kovats E. (1958). Gas-chromatographische charakteriserung organischer verbidungen. Teil 1: Retentions indices aliphatischer halogenide, Alkohole, Aldehyde und Ketone. Helvetica Chimica Acta: 41(7).

7. Lilia A. Conde-Hernández, José Á. Guerrero-Beltrán. (2014). Total phenolics and antioxidant activity of Piper auritum and Porophyllum ruderale. Food Chemistry 142, p455-460. 
8. Loayza L., Walter G., Lorenso D.; Dellacassa E., Mondello L., Dugo G. (2000). Composition of the essential oil of Pororophyllum ruderale(Jacq.) Cass. From Bolivia. Flavour and Fragrance Journal.14(6); p393-398.

9. Paolini J.(2005). Caractérisation des huiles essentielles par CPG/Ir, CPG/SM(IE et IC) et RMN du carbone-13 de Cistus albidus et de deux Asteraceae endémiques de Corse : Eupatorium cannabinum subsp. corsicum et Doronicum corsicum.. Chimie. Université de Corse. p343.

10. Raggi L.,CordeiroI.,Moreno P.R.H, and Young M.C.M. (2014). Essential oil composition from Porophyllum ruderale (Jacq.) Cass. subspecies, cultivated in two biogeographically different regions, Brazilian Cerrado and Atlantic Rain Forest. Planta Medica, 80(16)

11. RaggiL.,Young M.C.M, Cordeiro I and Moreno P.R.H. (2014). Differentiation of two Porophyllum ruderale (Jacq.) Cass. subspecies by the essential oil composition. Journal of Essential Oil Research ,27(1); P 30-33

12. Rondón, M.E.; Delgado, J.; Velaco, J.; Rojas, J.; Rojas, L.B.; Morales, A.; Carmona, J. (2008). Chemical composition and antibacterial activity of the essential oil from aerial parts of Porophyllum ruderale (Jacq.) Cass.collected in Venezuela. Ciência ,16, p5-9.

13. Takahashi H.T., NovelloC.R.,Ueda-Nakamura T., Filho B.P.D., Palazzo de Mello J.C. and Nakamura C.V. (2011).Thiophene Derivatives with Antileishmanial Activity Isolated from Aerial Parts of Porophyllum ruderale(Jacq.)Cass. Molecules, 16(5), p3469-3478. 